\title{
Oxandrolone Enhances Hepatic Ketogenesis in Adult Men
}

\author{
Gloria Lena Vega, PhD, ${ }^{*} \dagger$ Jacob J. Clarenbach, MD, ${ }^{*}$ Fredrick Dunn, MD, $\dagger$ \\ and Scott M. Grundy, MD, PhD*†
}

\section{ABSTRACT}

Background: Immediate administration of oxandrolone markedly increases hepatic lipase activity and reduces levels of plasma high-density lipoprotein.

Rationale for the study: We postulated that oxandrolone should increase hepatic lipase and that the nonesterified fatty acids generated would enhance hepatic ketogenesis during an extended fat tolerance test.

Main Results: Eighteen men participated in the study using short-term administration of oxandrolone $(10 \mathrm{mg} / \mathrm{d})$ over a week. Subjects had evaluation of hepatic ketogenesis at baseline and after 7 days of administration of oxandrolone. Ketogenesis was assessed by measuring plasma levels of 3-hydroxybutyrate during a fat tolerance test. Oxandrolone increased fasting levels of 3-hydroxybutyrate by $70 \%$, and increased the area under the curve during an FFT by $53 \%$ above pretreatment levels without affecting the areas under the curve for nonesterified fatty acids, glycerol, or triglycerides. Fasting 3-hydroxybutyrate levels correlated with nonesterified fatty acids and with triglycerides; however, there were no significant correlations with any other parameter.

Conclusions: This study shows that short-term administration of oxandrolone results in marked increases in hepatic ketogenesis. This finding is consistent with an increased influx of fatty acids into the liver secondary to lipoprotein lipolysis by increased hepatic lipase. However, the possibility cannot be ruled out that oxandrolone acts directly in the liver to stimulate fatty acid oxidation. Therefore, the observation of increased ketogenesis will require further studies to determine the molecular basis of the response.

Key Words: steroids, 3-hydroxybutyrate, nonesterified fatty acids, hepatic lipase

From the *Center for Human Nutrition of the University of Texas Southwestern Medical Center at Dallas, and the †Veterans Affairs Medical Center, Dallas, TX.

The study was supported partially by the Veterans Affairs Medical Center Merit Award, Moss Heart Foundation and Center for Human Nutrition.

Reprints: Gloria Lena Vega, PhD, and Scott M. Grundy, MD, PhD, Center for Human Nutrition, UT Southwestern Medical Center, 5323 Harry Hines Blvd., Dallas, TX 75390-9052. E-mail: Gloria.Vega@ utsouthwestern.edu and Scott.Grundy@utsouthwestern.edu.

\section{- INTRODUCTION}

Anabolic steroids stimulate expression of hepatic lipase (HL). ${ }^{1,2}$ Hepatic lipase possesses both phospholipase and triglyceride-lipase activities. An increase in HL activity is accompanied by a reduction in plasma highdensity lipoprotein cholesterol (HDL-C) levels; this increase further appears to enhance the lipolysis of triglyceride-rich lipoproteins (TGRLP) and to promote uptake of remnant lipoproteins into the liver. ${ }^{2-4}$ Because of the effects of anabolic steroids on HL, we posed the question of whether an increased lipolysis of TGRLP at the surface of liver cells might increase the influx of fatty acids into the liver. As a first step to examine this possibility, we further asked whether anabolic steroids might promote hepatic ketogenesis, which could be secondary to increased influx of fatty acids into the liver and enhanced fatty acid oxidation. Because a precise quantification of fatty acid oxidation in humans is not possible, we measured plasma levels of 3-hydroxybutyrate as a surrogate indicator. Previous studies have shown that plasma 3-hydroxybutyrate correlates with rates of ketogenesis, which in turn correlates with rates of fatty acid oxidation. ${ }^{5-8}$ If hepatic ketogenesis during anabolic steroid administration is not increased, then an increased influx of fatty acids into the liver is unlikely.

\section{- METHODS}

Eighteen adult men were recruited into the study at the Veterans Affairs Medical Center in Dallas. Their characteristics are shown in Table 1. Nine subjects had metabolic syndrome as previously defined. ${ }^{9}$ Basal plasma triglycerides ranged from $51 \mathrm{mg} / \mathrm{dL}$ to $340 \mathrm{mg} / \mathrm{dL}$, low-density lipoprotein cholesterol level ranged from $80 \mathrm{mg} / \mathrm{dL}$ to $182 \mathrm{mg} / \mathrm{dL}$ and HDL-C from $19 \mathrm{mg} / \mathrm{dL}$ to $74 \mathrm{mg} / \mathrm{dL}$. None were taking lipid-lowering drugs, and none had history of cardiovascular disease, endocrine disorders, liver dysfunction, or contraindications for participating in the study. The trial had a sequential design of baseline evaluation followed by oral administration of oxandrolone $(10 \mathrm{mg} / \mathrm{d})$ for 7 days with a repeat of the baseline tests. The protocol was approved by the Institutional Review Board for Investigation in Humans and all subjects gave informed written consent. 
TABLE 1. Clinical Characteristics of Subjects

\begin{tabular}{lcc}
\hline & Mean \pm SEM & Median \\
\hline Age (yr) & $58.6 \pm 1.8$ & 59.5 \\
Body mass index $\left(\mathrm{kg} / \mathrm{m}^{2}\right)$ & $29.8 \pm 1.2$ & 30.5 \\
Waist circumference $(\mathrm{cm})$ & $101.2 \pm 2.9$ & 99.8 \\
Systolic blood pressure $(\mathrm{mm} \mathrm{Hg})$ & $136.5 \pm 2.5$ & 135.0 \\
Diastolic blood pressure (mm Hg) & $79.6 \pm 1.9$ & 82.0 \\
Total cholesterol (mg/dL) & $202 \pm 7.8$ & 199 \\
Non-HDL cholesterol (mg/dL) & $163 \pm 8.9$ & 166 \\
Total apolipoprotein B (mg/dL) & $125 \pm 7.0$ & 132 \\
\hline HDL, high-density lipoprotein. & &
\end{tabular}

Subjects had a clinical evaluation for inclusion into the study, and after recruitment, they had anthropometry, measurement of fasting plasma lipids and lipoprotein cholesterol, and study of ketogenesis during an extended fat tolerance test (FTT). For the latter, after an overnight fast, subjects ingested a heavy whipping cream drink containing $75 \mathrm{~g}$ of fat $(100 \%$ of calories from fat with $70 \%$ long-chain saturates). After cream ingestion, they were allowed to drink water and sugar-free tea during the succeeding 10 hours. Arterialized blood samples were obtained in sodium-ethylenediaminetetraacetic acid $(2 \mathrm{mg} / \mathrm{mL})$ before $(t=0)$ and every 2 hours until 8 hours after the meal. Subjects kept their hand in an isothermal box $\left(T=70^{\circ} \mathrm{C}\right)$ to obtain arterialized blood samples. To avoid ongoing in vitro lipolysis by plasma lipoprotein lipase, blood samples were immediately placed on ice, centrifuged to separate plasma, which
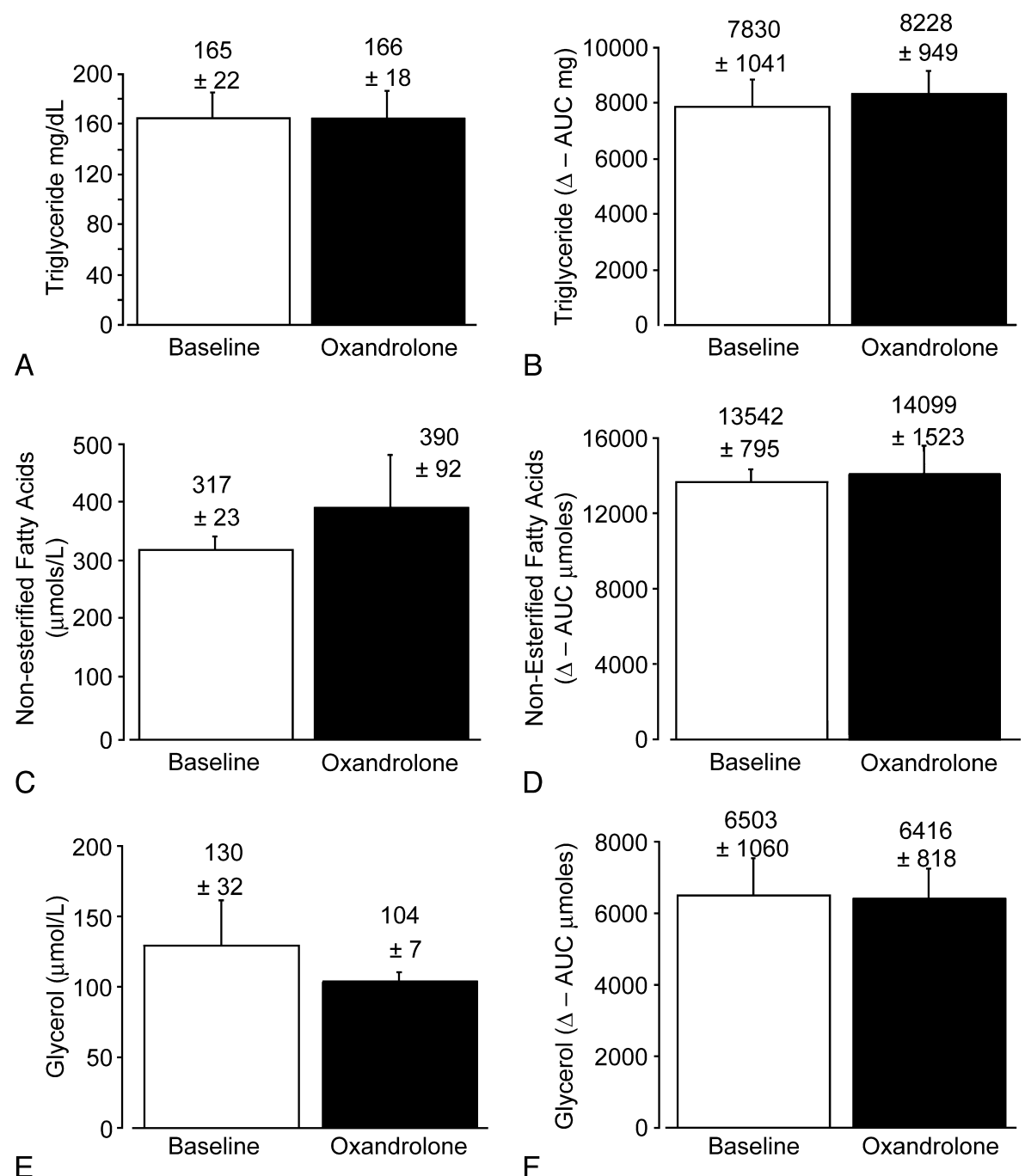

$E$

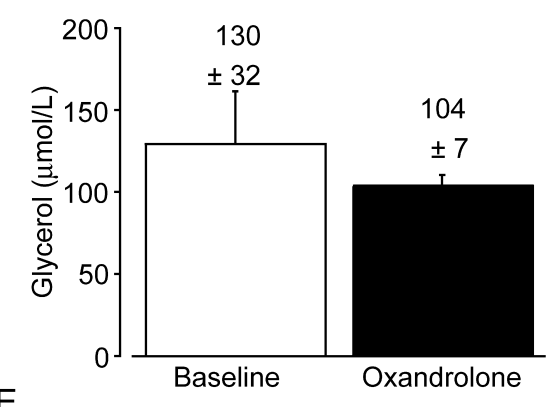

FIGURE 1. Effect of oxandrolone on plasma fasting levels and post-prandial levels of triglyceride (panels A and B, respectively), non-esterified fatty acids (panels $C$ and $D$, respectively) and glycerol (panels $E$ and $F$, respectively). The term baseline signifies the study before oxandrolone treatment and oxandrolone during treatment. There were no significant changes in either fasting or the post-prandial levels for any of the metabolites between baseline and on-treatment measurements. Numerical data shown in the figure represent means \pm standard error of the means. 


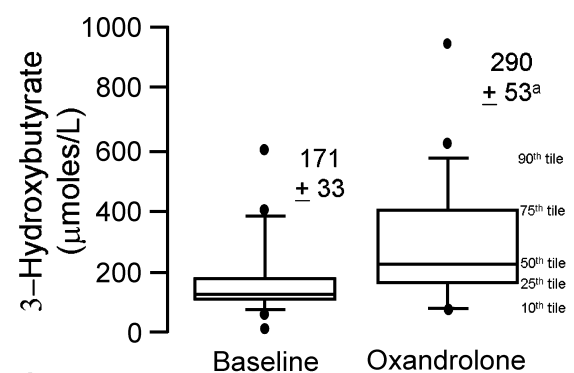

A

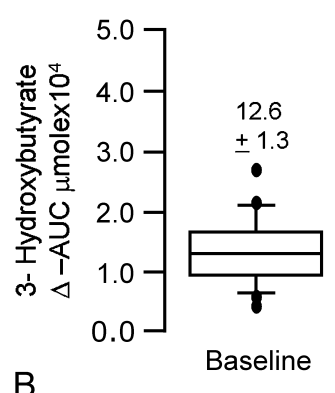

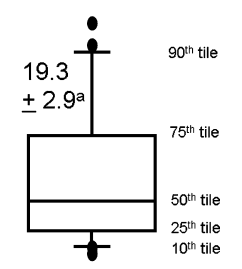

Oxandrolone

FIGURE 2. Effect of oxandrolone on fasting levels of 3-hydroxybutyrate (panel A) and during an extended fat tolerance test (panel B). Results are shown as box-and-whisker plots. Numerical data shown in the figure represent means \pm standard error of the means. The term baseline signifies the study before oxandrolone treatment and oxandrolone during treatment. Oxandrolone therapy significantly increased the fasting and post-prandial levels of 3-hydroxybutyrate by repeated ANOVA after Bonferroni-Dunn adjustments for multiplicity of testing $(P<0.0028$ for panel $\mathrm{A}$ and $P<0.0086$ for panel $\mathrm{B})$.

was frozen at $-80^{\circ} \mathrm{C}$. Analysis was carried out in less than 24 hours. In preliminary testing, we demonstrated that no significant lipolysis of triglycerides occurred before analysis under these conditions. The 3-hydroxybutyrate, nonesterified fatty acids (NEFA), triglycerides, and glycerol were measured from plasma spectrophotometrically using enzymatic assays (Roche Diagnostics/Boehringer Mannheim Corp, Indianapolis IN). Levels of plasma total cholesterol, triglycerides, and HDL-C were measured using standardized enzymatic assays as described previously. ${ }^{10}$ Levels of plasma apolipoprotein B were quantified as detailed previously. ${ }^{11}$

Data are summarized as mean \pm SEM. The primary end point of the study was the change in the area under the curve (AUC) of plasma 3-hydroxybutyrate during the extended FTT before and after 7 days of treatment with oxandrolone. The effects of oxandrolone on metabolites of interest were analyzed by comparing the baseline to treatment levels during fasting or the AUCs during the extended FTT. Analysis was carried out by repeatedmeasures analyses of variance with Bonferroni-Dunn adjustments for multiplicity of testing. Areas under the curve were calculated by the trapezoid formula. The StatView (version 5.0.1) program from SAS was used in the analyses of the data.

\section{RESULTS}

During oxandrolone treatment, the men had the expected marked lowering of HDL-C $(39 \pm 3$ [SEM] $\mathrm{mg} / \mathrm{dL}$ vs $26 \pm 2 \mathrm{mg} / \mathrm{dL}[P<0.001])$. Low-density lipoprotein cholesterol levels were unchanged $(131 \pm 7$ $\mathrm{mg} / \mathrm{dL}$ vs $136 \pm 8 \mathrm{mg} / \mathrm{dL}$ ). As shown in Figure 1, oxandrolone therapy produced no changes in either fasting levels or postprandial AUCs for plasma triglycerides, glycerol, or NEFA. In contrast, fasting levels of 3-hydroxybutyrate during oxandrolone treatment were increased by $70 \%$ (Fig. 2), and oxandrolone raised the AUC for 3-hydroxybutyrate by $53 \%$ after oral fat loading. There was a consistent direction of change in 3-hydroxybutyrate on oxandrolone therapy in spite of considerable baseline variation in NEFA levels among individuals of the study. This resulted in a highly statistically significant increase in 3-hydroxybutyrate levels on oxandrolone treatment (Fig. 2).

\section{DISCUSSION}

In the current study, oxandrolone therapy was accompanied by a striking increase in plasma 3-hydroxybutyrate levels. This change almost certainly reflects an increase in hepatic ketogenesis ${ }^{5-8}$ and raises the question of mechanism. Several possibilities can be considered.

First, an increased hepatic ketogenesis could be secondary to increased influx of fatty acids into the liver associated with an increased activity of HL. In fact, as previously reported, ${ }^{1,2,12}$ we observed a marked decrease in plasma levels of HDL-C associated with anabolic steroid administration. In the current study, the reduction in HDL-C was uniform, averaging about 33\%. Reductions in HDL-C levels occurred in subjects who even had a low baseline HDL before oxandrolone. Based on previous studies, ${ }^{1,2,12}$ we assume that the further reduction in HDL-C levels during oxandrolone administration was the consequence of an increase in HL activity. An increase in lipolytic activity at the surface of the hepatocytes could result in greater influx of fatty acids into the liver. Potential lipoprotein substrates for HL activities have been studied but have not been entirely resolved. High-density lipoprotein and TGRLP, including very low-density lipoprotein and chylomicrons and their remnants may be substrates. Most findings are based on in vitro studies and do not provide definite information on what actually occurs at the surface to the liver cell. However, the potential for lipolysis and release of fatty 
acids from several types of lipoproteins exist. These fatty acids could be a source for fatty acid oxidation.

Another possible mechanism for increased fatty acid oxidation with oxandrolone therapy could be an enhanced uptake of circulating NEFA by the liver. Some investigators have shown that anabolic steroids modestly raise the activity of plasma lipoprotein lipase. ${ }^{13}$ However, in this study, there was no increase in either NEFA or glycerol in the postprandial state. This finding speaks against the possibility that oxandrolone enhanced peripheral lipolysis by LPL and increased availability of NEFA for hepatic uptake. Further, the lack of rise in fasting NEFA on oxandrolone therapy does not support the possibility that adipose tissue lipolysis was enhanced by the steroid. Conversely, the lack of fall in fasting NEFA on oxandrolone does not support an increased fatty acid oxidation in muscle that could produce enhanced NEFA uptake by muscle.

Another possibility that cannot be ruled out is that oxandrolone directly promoted fatty acid oxidation in the liver. Anabolic steroids are reported to have a myriad of metabolic actions. ${ }^{14}$ Some of these may impinge on factors regulating fatty acid oxidation. The latter is regulated largely by the flux of acyl groups into the mitochondria. The major factor affecting acyl flux is availability, as discussed previously, but other factors are known to influence fatty acids oxidation. They include concentrations of malonyl-CoA, carnitine acylcarnitine translocase I and II activities, the redox state of nicotinamide adenine dinucleotide (NAD+/NADH), and the activities of various nuclear receptors (eg, peroxisome proliferator-activated receptor- $\alpha$ ) and hormones (epinephrine, thyroid hormones, insulin and glucagon), among others. ${ }^{15-17}$ Thus, it is possible that anabolic steroids may directly influence rates of fatty acid oxidation independently of availability of acyl groups derived from increased influx of fatty acids into the liver. The findings of the current study thus should open new avenues to understanding of regulation of fatty acid oxidation.

In summary, short-term treatment with oxandrolone leads to marked hepatic ketogenesis during fasting and during oral fat loading in adult men. Because anabolic steroids are known to increase HL activities, which could enhance fatty acid influx into the liver, we were prompted to ask whether there might be an increased ketogenesis associated with oxandrolone therapy. In fact, this was observed, which is consistent with our hypothesis. Nevertheless, beyond the basic observation, the actual mechanism for enhanced ketogenesis by anabolic steroids must await more detailed in vitro and in vivo studies in various animals. For future studies, we suggest that consideration be given to the possibility that HL activity is a greater regulator of hepatic fatty acid influx than previously recognized.

\section{ACKNOWLEDGMENTS}

The authors thank Laura Caldwell, PAC, Rita Nemons, RN, Davood Rezaei, Research Pharmacist and Marjorie Whelan, RN, for their contribution. Biman Pramanik, Mathew Kreth, and Mathew Crutchley provided excellent technical support for the conduct of the study.

\section{- REFERENCES}

1. Kantor MA, Bianchini A, Bernier D, et al. Androgens reduce HDL2-cholesterol and increase hepatic triglyceride lipase activity. Med Sci Sports Exerc. 1985;17:462-465.

2. Vega GL, Gao J, Bersot TP, et al. The -514 polymorphism in the hepatic lipase gene (LIPC) does not influence androgen-mediated stimulation of hepatic lipase activity. J Lipid Res. 1998;39:1520-1524.

3. Connelly PW, Maguire GF, Lee M, et al. Plasma lipoproteins in familial hepatic lipase deficiency. Arteriosclerosis. 1990;10:40-48.

4. Shafi S, Brady SE, Bensadoun A, et al. Role of hepatic lipase in the uptake and processing of chylomicron remnants in rat liver. J Lipid Res. 1994;35:709-720.

5. Garber AJ, Menzel PH, Boden G, et al. Hepatic ketogenesis and gluconeogenesis in humans. J Clin Invest. 1974;54:981-989.

6. Reichard GA Jr, Owen OE, Haff AC, et al. Ketone-body production and oxidation in fasting obese humans. $J$ Clin Invest. 1974;53:508-515.

7. Reichard GA Jr, Haff AC, Skutches CL, et al. Plasma acetone metabolism in the fasting human. $J$ Clin Invest. 1979;63:619-626.

8. Hall SE, Wastney ME, Bolton TM, et al. Ketone body kinetics in humans: the effects of insulin-dependent diabetes, obesity, and starvation. J Lipid Res. 1984;25: 1184-1194.

9. Grundy SM, Cleeman JI, Daniels SR, et al. Diagnosis and management of the metabolic syndrome: an American Heart Association/National Heart, Lung, and Blood Institute Scientific Statement. Circulation. 2005;25;112: 2735-2752.

10. Vega GL, Chandalia M, Szczepaniak LS, et al. Metabolic correlates of nonalcoholic fatty liver in women and men. Hepatology. 2007;46:716-722.

11. Vega GL, Grundy SM. Quantitation of apolipoprotein B by chemical methods. Methods Enzymol. 1996;263: 63-82.

12. Tikkanen MJ, Nikkila EA. Regulation of hepatic lipase and serum lipoproteins by sex steroids. Am Heart J. 1987; 113:562-567.

13. Sorva R, Kuusi T, Taskinen MR, et al. Testosterone substitution increases the activity of lipoprotein lipase and hepatic lipase in hypogonadal males. Atherosclerosis. 1988;69:191-197. 
14. Bain J. The many faces of testosterone. Clin Interv Aging. 2007;2:567-576.

15. McGarry JD, Brown NF. The mitochondrial carnitine palmitoyltransferase system. From concept to molecular analysis. Eur J Biochem. 1997;244:1-14.
16. Eaton S. Control of mitochondrial beta-oxidation flux. Prog Lipid Res. 2002;41:197-239.

17. Beylot M. Regulation of in vivo ketogenesis: role of free fatty acids and control by epinephrine, thyroid hormones, insulin and glucagon. Diabetes Metab. 1996;22:299-304. 